\title{
Targeting YAP suppresses ovarian cancer progression through regulation of the PI3K/Akt/mTOR pathway
}

\author{
XIAO WEI $^{1 *}$, YIJUAN JIA ${ }^{2 *}$, HUA LOU ${ }^{1}$, JINGJING MA $^{1}$, QUANFU HUANG ${ }^{3}$, YUNCHONG MENG ${ }^{3}$,

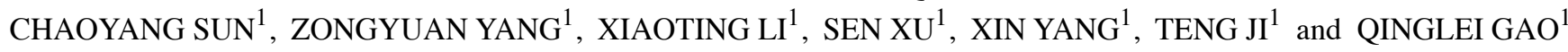 \\ ${ }^{1}$ Department of Obstetrics and Gynecology, Tongji Hospital, Tongji Medical College, \\ Huazhong University of Science and Technology; ${ }^{2}$ Department of Obstetrics and Gynecology, \\ Wuhan First Hospital; ${ }^{3}$ Department of Thoracic Surgery, Tongji Hospital, Tongji Medical College, \\ Huazhong University of Science and Technology, Wuhan, Hubei 430030, P.R. China
}

Received April 24, 2019; Accepted August 16, 2019

DOI: 10.3892/or.2019.7370

\begin{abstract}
Ovarian cancer (OC) is highly metastatic due to frequent peritoneal dissemination, and its treatment poses a major challenge in clinical practice. Yes-associated protein (YAP) is known to be associated with the development of multiple tumors. However, whether targeting YAP can restrain $\mathrm{OC}$ progression and the underlying mechanisms have yet to be fully elucidated. In the present study, YAP was found to be highly expressed in $\mathrm{OC}$, and its expression was correlated with the prognosis of $\mathrm{OC}$ patients. Moreover, silencing of YAP markedly inhibited the malignant behavior of OC cells, possibly through regulation of the PI3K/Akt/mTOR pathway. Notably, peptide 17, a YAP inhibitor, exerted a significant attenuating effect on OC progression by diminishing the activation of the PI3K/Akt/mTOR pathway in vitro as well as in vivo. Taken together, these findings demonstrated that targeting YAP attenuated OC progression and suggested the potential application of peptide 17 in OC therapy, thus providing new insights into improving the treatment of OC.
\end{abstract}

\section{Introduction}

Ovarian cancer (OC) remains a devastating gynecological malignancy in developed countries (1). The high mortality rate of this tumor is mainly due to the fact that the majority of the patients $(75 \%)$ present at an advanced stage and have already developed extensive peritoneal metastases (2). Patients often

Correspondence to: Dr Qinglei Gao, Department of Obstetrics and Gynecology, Tongji Hospital, Tongji Medical College, Huazhong University of Science and Technology, 1095 Jiefang Avenue, Wuhan, Hubei 430030, P.R. China

E-mail: qingleigao@hotmail.com.

${ }^{*}$ Contributed equally

Key words: ovarian cancer, Yes-associated protein, phosphoinositide 3-kinase/Akt/mammalian target of rapamycin, peptide 17 have non-specific pelvic or abdominal symptoms. Despite improvements in surgical techniques and treatment strategies for OC patients in recent years, the prognosis remains poor (3). Thus, it is crucial to explore novel targets for OC therapy.

The Hippo signaling pathway has been identified as a tumor suppressor pathway that is involved in cell death, cell proliferation and tissue growth (4). Yes-associated protein (YAP), a direct downstream effector of the Hippo pathway, is a key transcriptional co-activator that interacts with the TEA domain family member (TEAD) to regulate the expression of target genes $(5,6)$. Recent advances have revealed the upregulation of YAP in several types of cancer, such as breast cancer (7), hepatocellular carcinoma (HCC) (8) and lung adenocarcinoma (9), and have identified YAP as an oncogene in OC $(10,11)$. Moreover, it was demonstrated that YAP could form autocrine loops with the ERBB pathway to regulate OC initiation and progression (11). However, the effect and specific mechanism of targeting YAP in $\mathrm{OC}$ progression requires further investigation.

The aim of the present study was to determine the expression of YAP in OC tissues and its correlation with OC prognosis, as well as elucidate the underlying mechanism, hoping to uncover a novel approach to OC therapy.

\section{Materials and methods}

Cell culture. The human ovarian cancer cell lines ES-2 (cat. no. CRL-1978) and SK-OV-3 (cat. no. HTB-77), were purchased from the American Type Culture Collection (ATCC). All cell lines were tested every 6 months, or new ATCC cell lines were obtained every 6 months. SK-OV-3 and ES-2 were maintained in McCoy's $5 \mathrm{~A}$ medium supplemented with $10 \%$ fetal bovine serum and $1 \%$ antibiotic mixture $(10,000 \mathrm{U} / \mathrm{ml}$ penicillin and $10 \mathrm{mg} / \mathrm{ml}$ streptomycin) and cultured with $5 \% \mathrm{CO}_{2}$ at $37^{\circ} \mathrm{C}$.

Reagents and antibodies. Negative control (siControl) and YAP-1-specific (siYAP-1, siYAP-2) siRNA were purchased from Invitrogen; Thermo Fisher Scientific, Inc. (sequences: siYAP-1: 5'-CAGCAGAAUAUGAUGAACUCGGCUU-3'; siYAP-2: 5'-GGAAGGAGAUGGAAUGAACAUAGAA-3'). Peptide 17 was purchased from Selleck Chemicals. YAP, phospho-Akt (Ser473), phospho-PI3K p85 (Tyr458)/p55 
(Tyr199), mTOR, and Akt2 antibody were obtained from Cell Signaling Technology. The GSK3- $\beta$ and GAPDH antibodies were obtained from Proteintech Group, Inc. The Ki-67, PI3K p85 beta, phospho-GSK3- $\beta$ (Ser9), and phospho-mTOR (Ser2448) antibodies were obtained from Abcam. Matrigel was purchased from BD Biosciences. The secondary antibodies were horseradish peroxidase (HRP)-conjugated goat anti-rabbit and anti-mouse IgG (1:5,000; Antgene). The complete information on all the antibodies used in the present study is provided in supplementary Table SI.

Migration assay. Transwell plates ( $8-\mu \mathrm{m}$ pore size; Corning, Inc.) were used for the migration assay as described previously (12). Cells transfected with siControl or siYAP or treated with peptide $17(250 \mathrm{nM})$ were harvested and re-suspended with serum-free McCoy's 5A and seeded at a cell density of $2 \times 10^{4}$ into the upper chamber. Complete media $(500 \mu \mathrm{l})$ were added to the bottom chambers. The cells were incubated at $37^{\circ} \mathrm{C}$ for $24 \mathrm{~h}$. After 1 day of incubation, the cells that migrated to the lower surface of the membrane were fixed with $4 \%$ paraformaldehyde for $15 \mathrm{~min}$, then washed with phosphate-buffered saline (PBS) for 3 times and stained with $0.05 \%$ crystal violet solution for $15 \mathrm{~min}$. Non-migrating cells on the top surface of the filter were carefully wiped off with a cotton swab, and the migrating cells were quantified by counting five random fields using a phase contrast microscope.

Invasion assay. In vitro cell invasion was investigated using a Transwell assay (8.0 $\mu \mathrm{m}$, Corning, Inc.) coated with basement membrane Matrigel (BD Biosciences). Cells transfected with siControl or siYAP or treated with peptide $17(250 \mathrm{nM})$ were harvested and seeded at a cell density of $2 \times 10^{4}$ into the upper chamber. Complete media $(500 \mu \mathrm{l})$ were added to the bottom chambers. After $48 \mathrm{~h}$ of incubation, non-invading cells on the top surface of the filter were carefully wiped off with a cotton swab, and the invading cells were quantified by counting five random fields using a phase contrast microscope.

Wound healing assay. Cells transfected with siControl or siYAP or treated with peptide $17(250 \mathrm{nM})$ were seeded in 6-well plates in triplicate. After the cells had reached $\sim 100 \%$ confluence, the monolayers were manually scratched using a sterile $10-\mu 1$ pipette tip and cultured for $24 \mathrm{~h}$. The area of the scratch was photographed under a microscope (Olympus Corporation) and measured using ImageJ software (National Institutes of Health). The results were expressed as a percentage of the original area.

Cell viability assay. Cell viability was determined using a Cell Counting Kit-8 (CCK-8; Dojindo Molecular Technologies, Inc.) at defined intervals. Cells transfected with siControl or siYAP $\left(4 \times 10^{3}\right.$ cells per well) were placed in 96 -well plates in triplicate and incubated for $72 \mathrm{~h}$. After treatment, cell viability was detected using the CCK-8 assay according to the manufacturer's instructions. After incubating with CCK- 8 dye at $37^{\circ} \mathrm{C}$ for $2 \mathrm{~h}$ in the dark, the absorbance value was measured by SpectraMax190 (Molecular Devices, LLC) at a wavelength of $450 \mathrm{~nm}$.

EdU assay. The EdU assay was carried out using the Cell-Light ${ }^{\mathrm{TM}}$ EdU imaging detecting kit according to the manufacturer's instructions (RiboBio).
Immunohistochemistry (IHC). Normal ovaries, normal fallopian tubes and OC tissues were collected from patients undergoing surgery at the Department of Gynecological Oncology of Tongji Hospital (Wuhan, China). IHC staining was conducted as previously described $(12,13)$.

Gene expression and reverse transcription-quantitative polymerase chain reaction $(R T-q P C R)$ analysis. Total RNA was isolated from cells or tissues using TRIzol Reagent (Invitrogen; Thermo Fisher Scientific, Inc.) according to the manufacturer's protocols. NanoDrop (Thermo Fisher Scientific, Inc.) was used to assess the quality and quantity of the total RNA. Total RNA (1 $\mu \mathrm{g})$ was used to synthesize cDNA using M-MLV reverse transcriptase (Takara Bio, Inc.). RT-qPCR detection was performed using the Bio-Rad CFX96 system with SYBR-Green to determine the expression level of mRNA of interest (Bio-Rad Laboratories, Inc.). The primers used were as follows: GAPDH forward, 5'-ATGGAAATC CCATCACCATCTT-3' and reverse, 5'-CGCCCCACTTGA TTTTGG-3'; YAP forward, 5'-TAGCCCTGCGTAGCCAGT TA-3' and reverse, 5'-TCATGCTTAGTCCACTGTCTGT-3'. The comparative $\mathrm{Cq}$ method was used to calculate the relative mRNA expression levels. The expression level of GAPDH was used as a loading control.

Western blot analysis. After washing twice with cold PBS, the cells were lysed with radioimmunoprecipitation assay (RIPA) lysis buffer (Beyotime Institute of Biotechnology) containing 1X protease inhibitor cocktail (Roche Diagnostics). Proteins were separated and electrophoresed on $10 \%$ sodium dodecyl sulfate polyacrylamide gel electrophoresis gels, transferred to nitrocellulose membranes by a wet transfer apparatus, and then blocked with TBST buffer $(25 \mathrm{mmol} / 1 \mathrm{Tri}-\mathrm{HCl}, \mathrm{pH} 7.5$, $137 \mathrm{mmol} / 1 \mathrm{NaCl}, 2.7 \mathrm{mmol} / \mathrm{K} \mathrm{KCl}$ and $0.05 \%$ Tween-20) with $5 \%$ bovine serum albumin for $1 \mathrm{~h}$ at $37^{\circ} \mathrm{C}$. The blots were probed with the indicated antibodies in blocking buffer overnight at $4^{\circ} \mathrm{C}$. GAPDH was used as the internal control. After the membranes were washed with TBST 3 times for 20 min each time, HRP-linked secondary antibody (Antgene) was applied to the membrane for $60 \mathrm{~min}$. The protein was finally detected using enhanced chemiluminescence (ECL; Bio-Rad Laboratories, Inc.). Band density was measured by ImageJ software (National Institutes of Health) and normalized to GAPDH.

Mouse experiments. Six-week-old female BALB/c-nu mice ( $n=10$ weight: $17-19 \mathrm{~g}$ ) were obtained from the Shanghai Animal Experimental Center. All mice were housed under pathogen-free conditions in the Animal Research Center of Tongji Hospital at $22^{\circ} \mathrm{C}$ with 12-h light/dark cycles and free access to water and food. SK-OV3-ip3-luc $\left(1 \times 10^{6}\right)$ cells were injected orthotopically under the ovarian bursa. One week after surgery, the mice were randomized into the control (saline) and treatment (peptide 17) experimental groups $(n=5$ per group). Peptide 17 was administered i.p. $(0.2 \mathrm{mg} / \mathrm{kg})$ daily and saline was used as control. The mice were anesthetized with pentobarbital sodium $(75 \mathrm{mg} / \mathrm{kg}$ ) after 28 days of treatment, and imaged with the IVIS SPECTRUM system (Caliper; Xenogen) $10 \mathrm{~min}$ after i.p. injection of $100 \mathrm{mg} / \mathrm{kg}$ D-luciferin substrate. Living Image software version 4.3.1 (Caliper; Xenogen) was used for the analysis. The tumor-bearing mice 
A

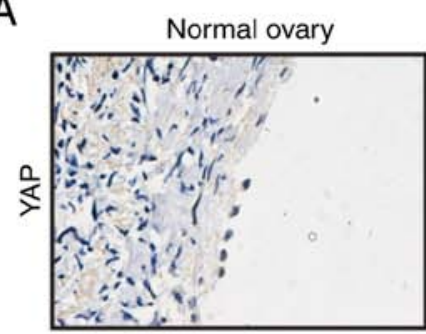

B

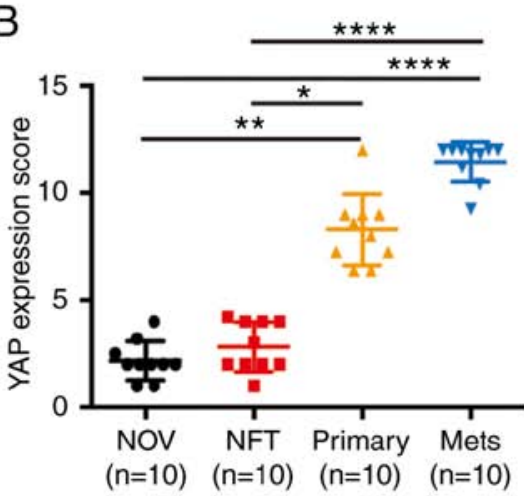

Normal fallopian tube

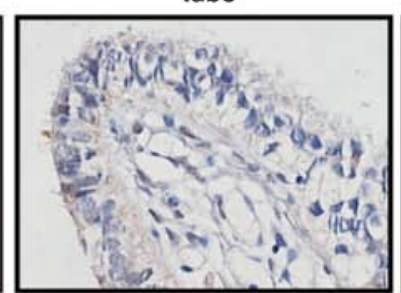

C

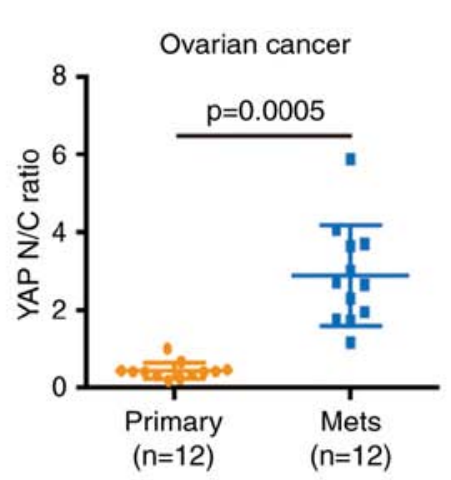

Primary

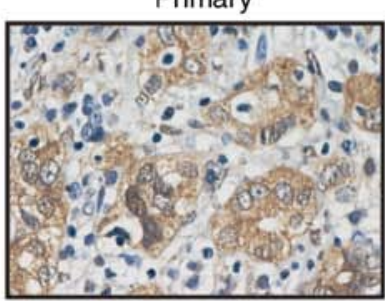

Metastases

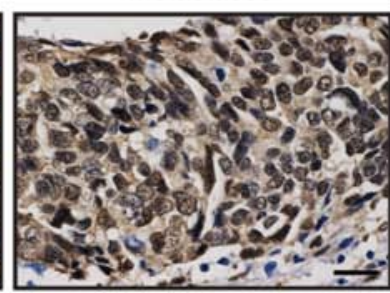

YAP PFS

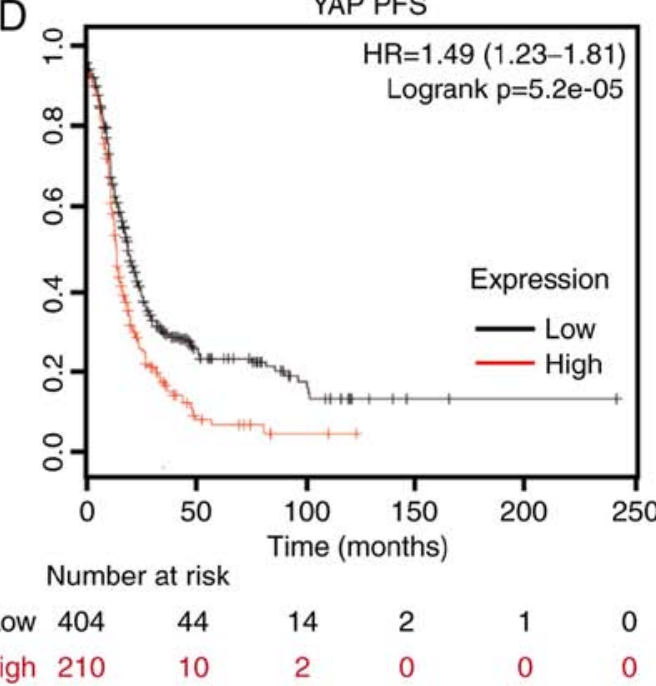

Figure 1. YAP is highly expressed in OC and is correlated with poor patient prognosis. (A) Representative YAP IHC image from analyses of human ovarian samples including normal ovaries, normal fallopian tubes, paired primary tumors and metastases of epithelial OC patients. Scale bar, $20 \mu$ m. (B) YAP expression scores of the above IHC-stained tissues: Normal ovaries (NOV), normal fallopian tubes (NFT), primary tumors (Primary), and metastases (Mets) ( $\mathrm{n}=10)$. (Kruskal-Wallis test, ${ }^{*} \mathrm{P}<0.05,{ }^{* *} \mathrm{P}<0.01,{ }^{* * * *} \mathrm{P}<0.0001$. (C) Distribution of nuclear $(\mathrm{N})$ to cytoplasmic (C) ratio (N/C ratio) of YAP staining in paired primary tumors (Primary) and metastases (Mets) of OC $(n=12)$. Geometric mean for N/C ratio for each category was calculated. (D) Progression-free survival (PFS) curve in OC patients with low or high expression levels of YAP. OC, ovarian cancer; IHC, immunohistochemistry; YAP, Yes-associated protein.

were sacrificed by cervical dislocation, and the tumors were removed for assessment.

Colony formation. Cells transfected with siControl or siYAP were seeded in 6-well plates and incubated at $37^{\circ} \mathrm{C}$ for 10 days. For the peptide 17 experiment, cells were plated in 6-well plates and treated with peptide $17(250 \mathrm{nM})$ for 10 days; the medium was replaced every $48 \mathrm{~h}$ to ensure constant levels of the drug. The cells were stained with $0.1 \%$ (w/v) crystal violet solution.

Data availability. The Kaplan-Meier plotter tool (http://kmplot. com/analysis/) was used to generate survival curves combining YAP1 (Affymetrix probe 224895_at) mRNA data from all public ovarian cancer datasets (14).

Statistical analysis. All data analyses in the present study were performed using GraphPad Prism 6 (GraphPad Software, Inc.). Data are expressed as the means \pm standard deviation from at least 3 independent experiments. Two experimental groups were compared using a paired Student's t-test for paired data or a Student's t-test for unpaired data. Analysis of variance was used to compare differences among multiple groups (post hoc test: Turkey's multiple comparisons test). Kruskal-Wallis test (post hoc test: Dunn's multiple comparisons test) was used to compare differences in YAP expression among normal ovaries, normal fallopian tubes, primary tumors and metastases. P-values $<0.05$ were considered to indicate statistically significant differences.

\section{Results}

YAP is significantly upregulated in human OC tissues and is correlated with poor prognosis. To explore the role of YAP in OC, YAP expression was first determined in normal ovaries, normal fallopian tubes and paired primary tumors and metastases of epithelial ovarian cancer (EOC) by IHC. The information on different tissues is provided in the supplementary Table SII. Compared with normal ovaries and normal fallopian tubes, YAP expression was significantly higher in OC primary tumors and metastases (Fig. 1A and B) (primary vs. normal ovary, $\mathrm{P}=0.0051$; primary vs. normal fallopian tube, $\mathrm{P}=0.0371$; metastases vs. normal ovary: $\mathrm{P}<0.0001$; metastases vs. normal fallopian tube, $\mathrm{P}<0.0001$ ). Notably, higher nuclear YAP levels were observed in metastases compared with primary tumors (Fig. 1C). Furthermore, Kaplan-Meier plot analysis of YAP expression in OC was performed to determine whether YAP is correlated with prognosis in OC patients. The result indicated an association of high YAP expression with poor progression-free survival (PFS) in OC patients (Fig. 1D). Thus, these results confirmed that YAP is upregulated in OC tissues and is correlated with 

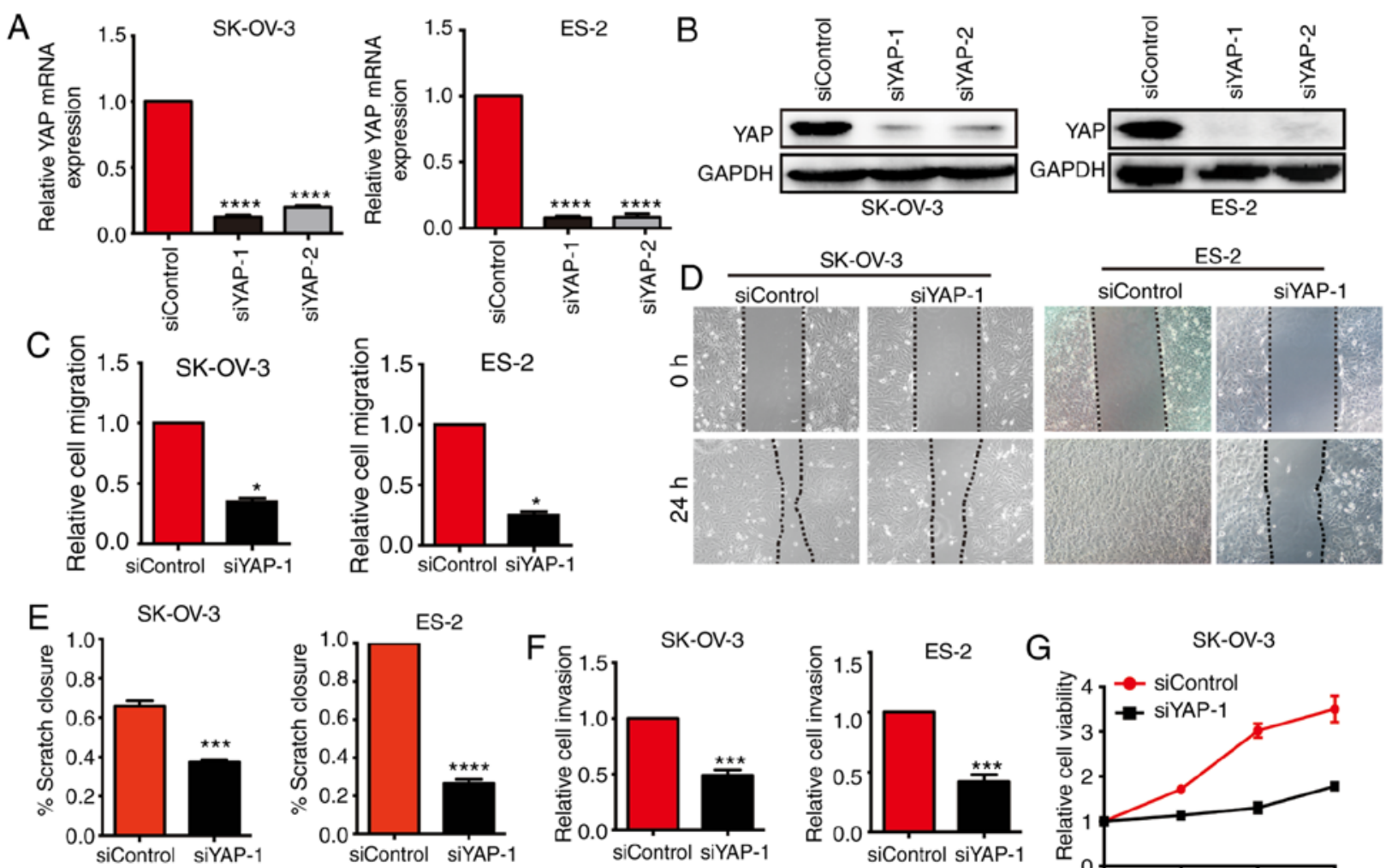

G
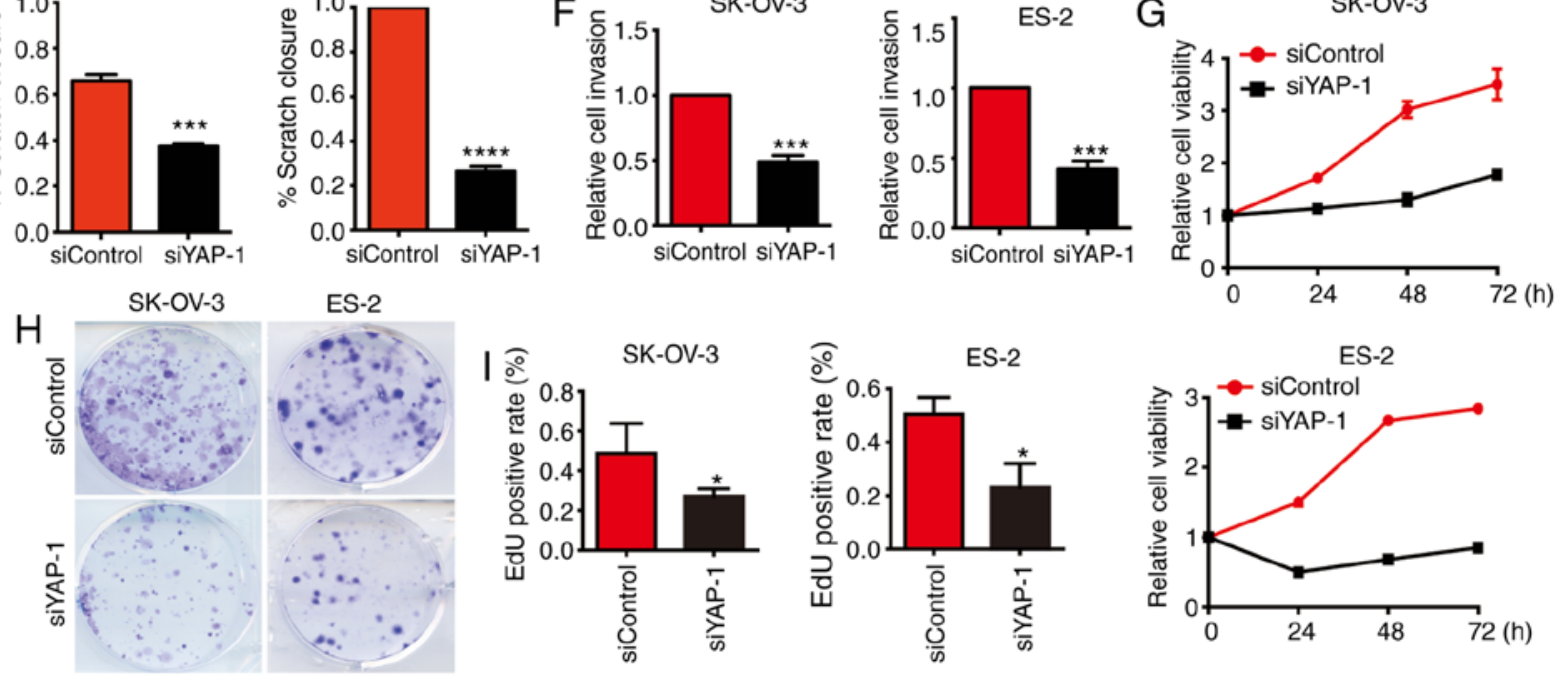

Figure 2. YAP silencing inhibits the malignant behavior of OC cells. (A) qPCR analysis detected the mRNA expression of YAP in SK-OV-3 and ES-2 cells transfected with siControl or siYAP-1 and siYAP-2. ${ }^{* * * *} \mathrm{P}<0.0001$. (B) Western blot analysis of the expression of YAP in SK-OV-3 and ES-2 cells transfected with siControl or siYAP-1 and siYAP-2. GAPDH was used as loading control. (C) The migratory ability of SK-OV-3 and ES-2 cells transfected with siControl or siYAP-1 was evaluated by Transwell assays and quantified. * $\mathrm{P}<0.05$. (D) Representative images and (E) statistical analysis of wound closure ability of SK-OV-3 and ES-2 cells transfected with siControl or siYAP-1 at 0 and 24 h. ${ }^{* * *} \mathrm{P}<0.001,{ }^{* * * *} \mathrm{P}<0.0001$. (F) The invasive ability of SK-OV-3 and ES-2 cells transfected with siControl or siYAP-1 was evaluated by Transwell assays and quantified. ${ }^{* * *} \mathrm{P}<0.001$. (G) Cell viability assays were performed to evaluate the proliferation of SK-OV-3 and ES-2 cells transfected with siControl or siYAP-1 at different time-points. (H) Colony formation ability of SK-OV-3 and ES-2 cells transfected with siControl or siYAP-1. (I) The proliferation of SK-OV-3 and ES-2 cells transfected with siControl or siYAP-1 was measured by EdU assay. Quantification of EdU-positive nuclei from $n=3$ experiments. ${ }^{*} \mathrm{P}<0.05$. YAP, Yes-associated protein; qPCR, quantitative polymerase chain reaction.

patient survival, suggesting that YAP may affect OC progression.

YAP silencing inhibits the malignant behavior of OC cells. To further explore the function of YAP in OC, two siRNAs targeting YAP and siControl were employed to transfect SK-OV-3 and ES-2 cells. qPCR and western blot analyses demonstrated that transfection of siYAP-1 and siYAP-2 efficiently inhibited YAP expression at both the mRNA and protein levels, particularly siYAP-1 (Fig. 2A and B). Transwell assays indicated that the migratory ability of both SK-OV-3 and ES-2 cells transfected with siYAP was markedly inhibited compared with cells transfected with siControl (Fig. 2C). Consistently, wound healing assays also confirmed that silencing of YAP diminished the migration of OC cells (Fig. 2D and E). We also demonstrated that YAP silencing inhibited the invasion of OC cells, as assessed by Transwell assays (Fig. 2F). Cell viability assay demonstrated that silencing of YAP inhibited the proliferation of OC cells (Fig. 2G). Similarly, colony formation assays confirmed that the colony-forming ability of OC cells was reduced upon transfection with siRNA of YAP (Fig. 2H). The EdU assay also supported these conclusions (Fig. 2I). Thus, the findings demonstrated that silencing of YAP diminished the migration, invasion and proliferation of $\mathrm{OC}$ cells, indicating that YAP may be a potential target for the treatment of OC.

YAP silencing regulates the PI3K/Akt/mTOR pathway. To further explore the mechanism through which silencing of YAP inhibits the malignant behavior of OC cells, we evaluated the activation of the PI3K/Akt/mTOR pathway, a key signaling pathway involved in tumor progression. The phosphorylation status of PI3K, Akt 
A

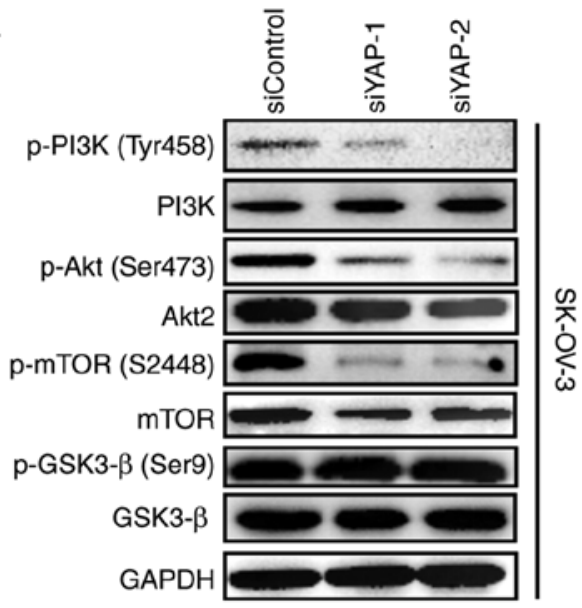

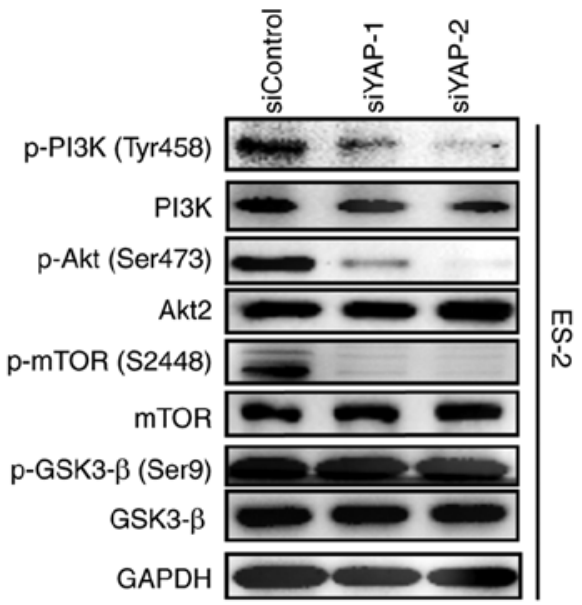

C
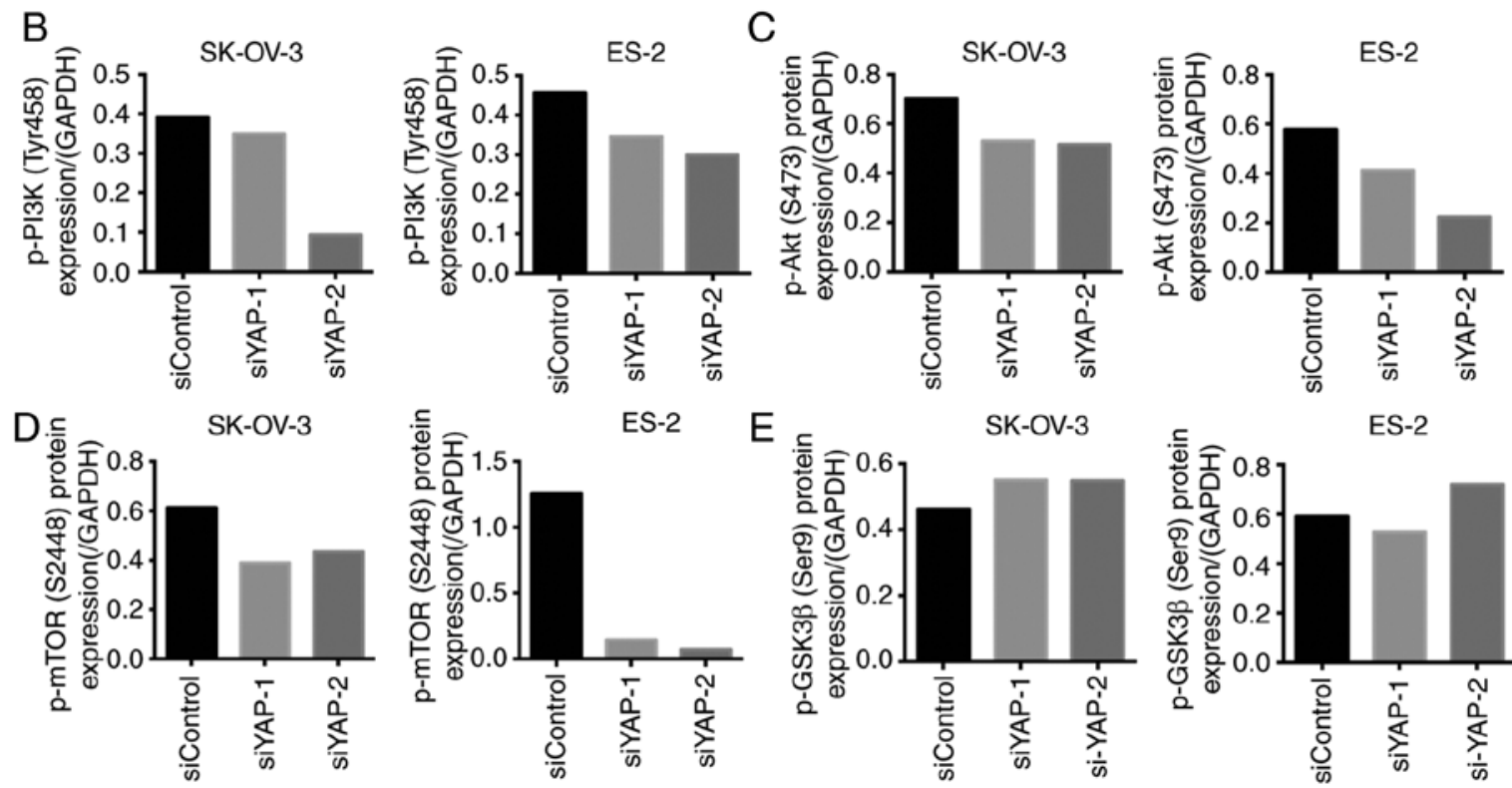

ES-2

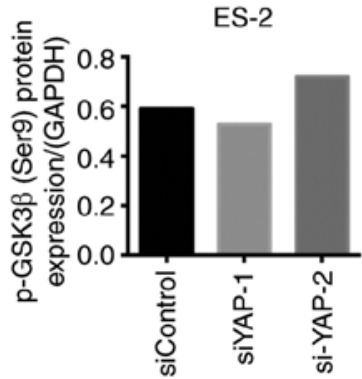

Figure 3. YAP silencing regulates the PI3K/AKT/mTOR pathway. (A) Western blot analyzed the expression of phospho-PI3K (Tyr458), phospho-Akt (Ser473), phospho-mTOR (S2448), phospho-GSK3- $\beta$ (Ser9), PI3K, mTOR, Akt2, and GSK3- $\beta$ in SK-OV-3 and ES-2 cells transfected with siYAP-1, siYAP-2, or siControl. GAPDH was used as a loading control. (B) Quantification of phospho-PI3K (Tyr458) relative to GAPDH in SK-OV-3 and ES-2 cells transfected with siYAP-1, siYAP-2, or siControl. (C) Quantification of phospho-Akt (Ser473) relative to GAPDH in SK-OV-3 and ES-2 cells transfected with siYAP-1, siYAP-2, or siControl. (D) Quantification of phospho-mTOR (S2448) relative to GAPDH in SK-OV-3 and ES-2 cells transfected with siYAP-1, siYAP-2, or siControl. (E) Quantification of phospho-GSK3- $\beta$ (Ser9) relative to GAPDH in SK-OV-3 and ES-2 cells transfected with siYAP-1, siYAP-2, or siControl. YAP, Yes-associated protein; GSK, glycogen synthase kinase.

and mTOR, the key signaling proteins of the PI3K/Akt/mTOR pathway, was assessed by western blotting. Compared with siControl-transfected cells, the phosphorylation of PI3K was significantly lower in siYAP-transfected SK-OV-3 and ES-2 cells (Fig. 3A and B). Similarly, downregulation of YAP expression decreased the phosphorylation of Akt and mTOR in both types of OC cells (Fig. 3A, C and D). Moreover, the activation of GSK-3 $\beta$ was assessed in both cell lines, since glycogen synthase kinase (GSK)-3 $\beta$ is another downstream regulator of the PI3K/Akt pathway. However, no significant changes in the phosphorylation status of GSK-3 $\beta$ were observed between siControl-transfected and siYAP-transfected cells (Fig. 3A and E). These results indicate that silencing of YAP may inhibit the activation of the $\mathrm{PI} 3 \mathrm{~K} / \mathrm{Akt} / \mathrm{mTOR}$ pathway, suggesting that this be the mechanism underlying YAP-regulated OC progression.

Peptide 17 inhibits the malignant behavior of OC cells and modulates the PI3K/Akt/mTOR pathway. As YAP silencing significantly inhibited the malignant behavior of OC cells, we next investigated whether peptide 17, a YAP inhibitor, exerted the same effect. Wound healing assays indicated that peptide 17 markedly impaired the migration of SK-OV-3 and ES-2 cells compared with the control group (Fig. 4A and B). Peptide 17 also inhibited the invasive ability of both SK-OV-3 and ES-2 cells, as evidenced by Transwell assays (Fig. 4C). The colony formation assay demonstrated that peptide 17 significantly inhibited colony formation by both SK-OV-3 and ES-2 cells (Fig. 4D). Similar results were also obtained by the EdU assay (Fig. 4E). These results demonstrated that peptide 17 inhibited the malignant behavior of OC cells. Considering that silencing YAP inhibited activation of the PI3K/Akt/mTOR pathway, we further investigated whether peptide 17 exerted a similar effect. Western blot analysis demonstrated that phosphorylation of PI3K, Akt and mTOR was lower in cells treated with peptide 17 compared with the control group (Fig. 4F). These results demonstrated 

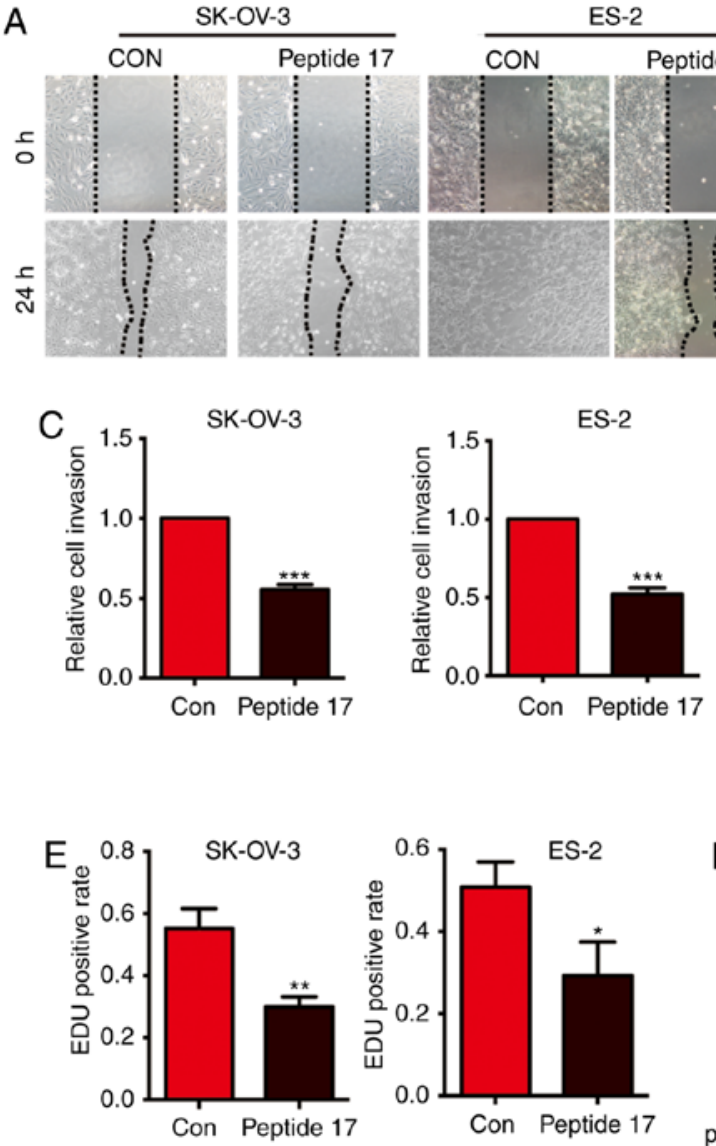
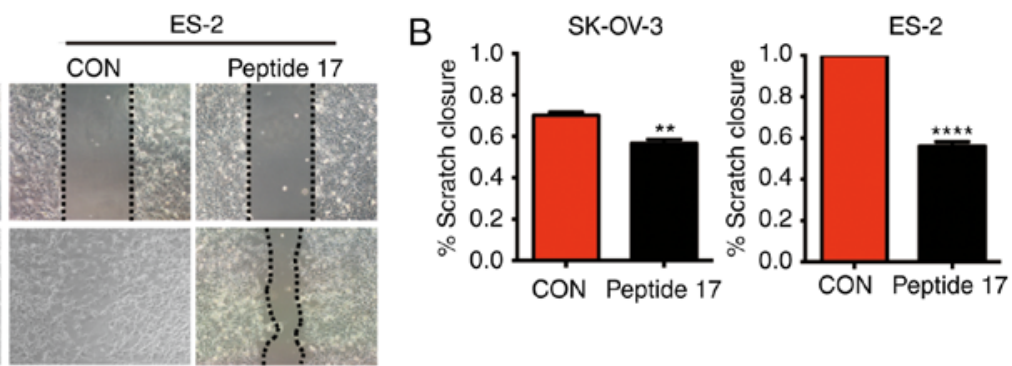

D

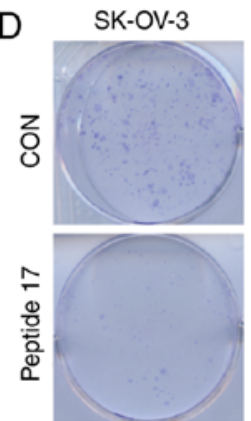

ES-2

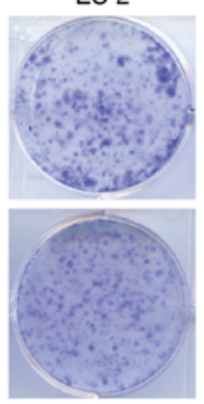

$\mathrm{F}$

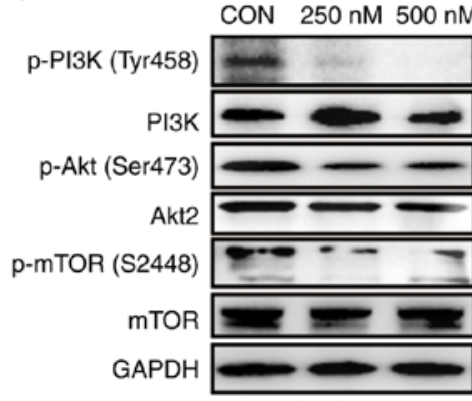

SK-OV-3

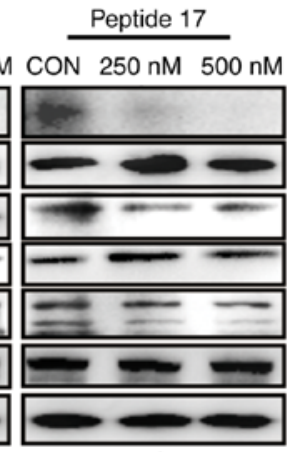

ES-2

Figure 4. Peptide 17 inhibits the malignant behavior of OC cells and modulates the PI3K/AKT/mTOR pathway. (A) Representative images from wound-healing assays using SK-OV-3 and ES-2 cells treated with or without peptide 17 at 0 and $24 \mathrm{~h}$. (B) The wound healing assay results are quantified in the histogram. ${ }^{* *} \mathrm{P}<0.01,{ }^{* * * * *} \mathrm{P}<0.0001$. (C) The invasive ability of SK-OV-3 and ES-2 cells treated with or without peptide 17 were evaluated by Transwell assays and quantified. ${ }^{* * *} \mathrm{P}<0.001$. (D) Colony formation of SK-OV-3 and ES-2 cells treated with or without peptide 17. (E) EdU assay was used to measure the proliferation of SK-OV-3 and ES-2 cells treated with or without peptide 17. Quantification of EdU-positive nuclei from $\mathrm{n}=3$ experiments. ${ }^{*} \mathrm{P}<0.05,{ }^{* *} \mathrm{P}<0.01 .(\mathrm{F})$ Western blotting was used to analyze the expression of phospho-PI3K (Tyr458), phospho-Akt (Ser473), phospho-mTOR (S2448), PI3K, mTOR, and AKT2 of SK-OV-3 and ES-2 cells treated with or without peptide 17 of various concentrations ( 250 and $500 \mathrm{nM})$. GAPDH was used as the loading control. OC, ovarian cancer.

that peptide 17 markedly attenuated the malignant behavior of OC cells and modulated the PI3K/Akt/mTOR pathway, suggesting that peptide 17 may be a potential therapeutic strategy for OC.

Peptide 17 restrains OC progression in vivo. Next, to verify whether inhibition of YAP with peptide 17 restrains OC progression in vivo, an orthotopic ovarian cancer model utilizing SK-OV-3-ip3-luc cells was employed. Consistent with the in vitro results, mice treated with peptide 17 developed significantly smaller tumors compared with mice treated with saline (Fig. 5A). To better understand these results, the tumors were removed at the end of the experiment, and further experiments were conducted. Subsequently, tumors from peptide 17-treated mice exhibited increased Ki-67 staining, as evidenced by IHC, compared with those in the saline group (Fig. 5B), indicating that peptide 17 inhibited the growth of OC in vivo. Since it was determined that peptide 17 could regulate the activation of the $\mathrm{PI} 3 \mathrm{~K} / \mathrm{Akt} / \mathrm{mTOR}$ signaling pathway in OC cells, phospho-Akt
(Ser473) and phospho-mTOR (S2448) expression were detected in the obtained tumor sections. Mechanistically, IHC analysis revealed significantly diminished staining of phospho-Akt (Ser473) (Fig. 5C) and phospho-mTOR (S2448) in peptide 17-treated mice, compared with the saline group (Fig. 5D). As shown by western blot analysis, the expression of phospho-PI3K (Tyr458), phospho-Akt (Ser473) and phospho-mTOR (S2448) was found to be markedly impaired in tumors from peptide 17-treated mice compared with those from saline-treated mice (Fig. 5E-F). Thus, these data demonstrated that peptide 17 significantly attenuated OC progression through inhibition of the PI3K/Akt/mTOR signaling pathway.

\section{Discussion}

In the present study, the expression of YAP in OC was determined, and a correlation between YAP expression and OC prognosis was identified. Furthermore, YAP silencing was shown to inhibit malignant phenotypes of OC cells through regulation of the PI3K/Akt/mTOR pathway. Of note, peptide 17, 
A
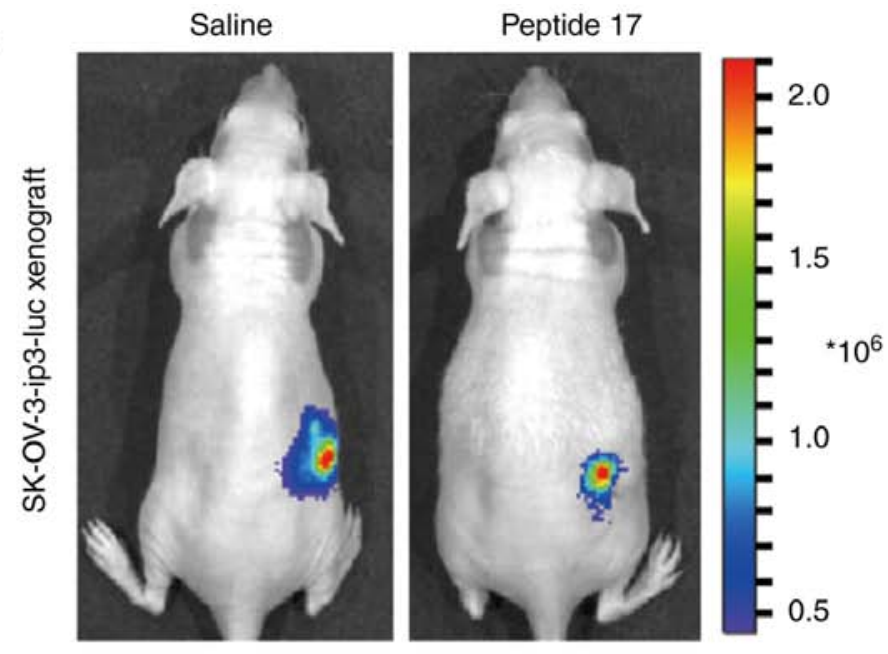

Radiance $\left(\mathrm{p} / \mathrm{sec} / \mathrm{cm}^{2} / \mathrm{sr}\right)$
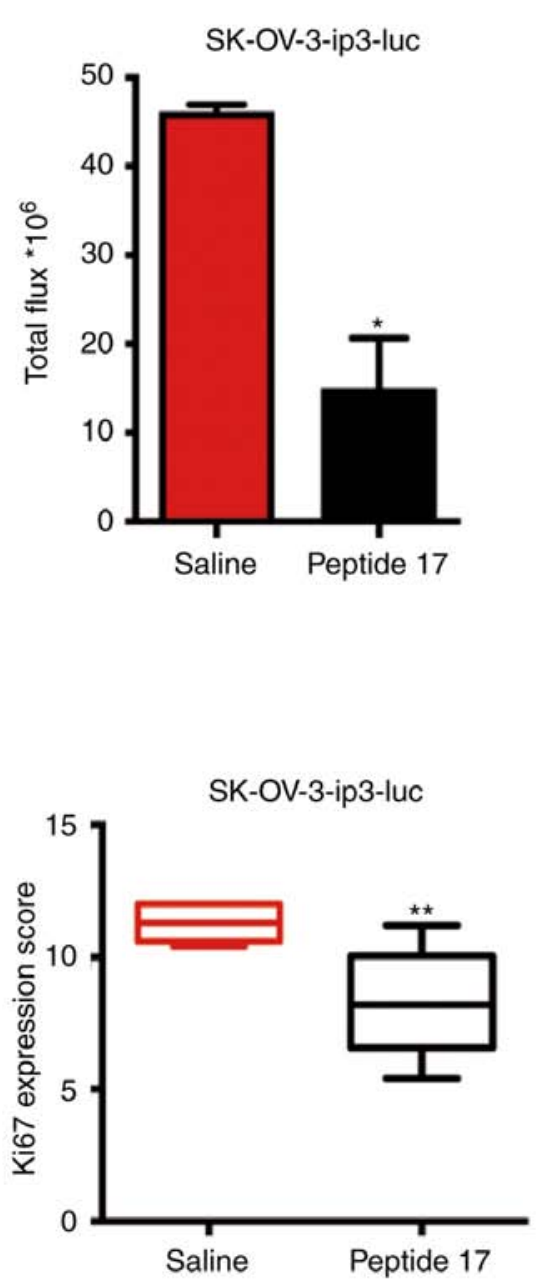

B
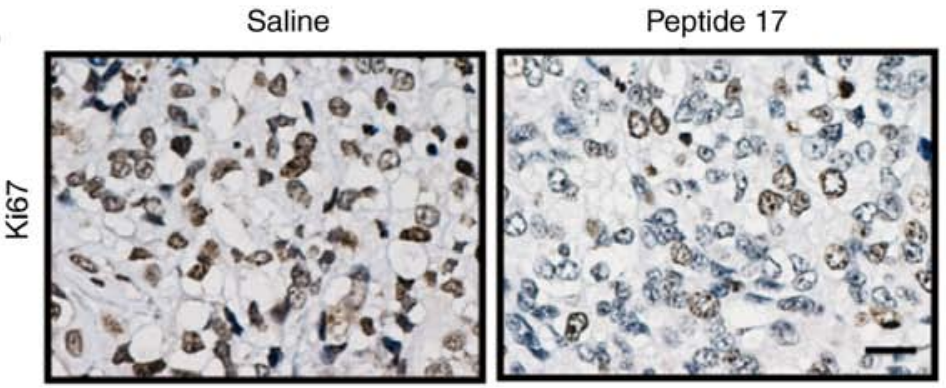

C

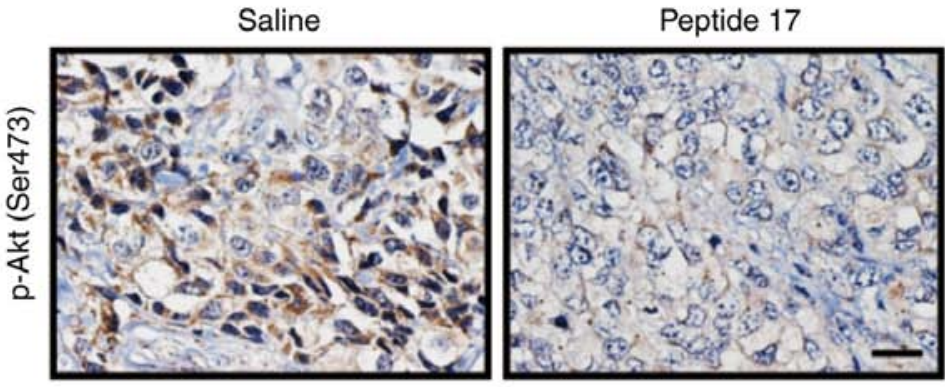

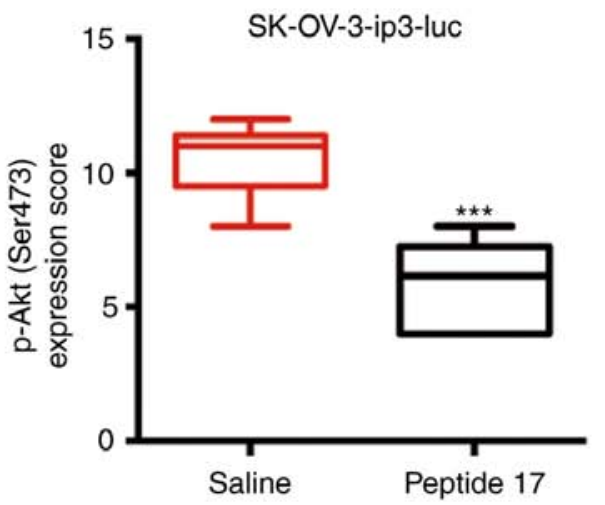

Figure 5. Peptide 17 restrains OC progression in vivo. (A) Representative bioluminescence images (left) and quantification (right) of mice (n=5 per group) treated with saline or peptide 17 for 4 weeks. Treatment was given after 1 week of SK-OV3-ip3-luc cells were injected orthotopically under the ovarian bursa ${ }^{*} \mathrm{P}<0.05$. (B) Representative images (left) and quantification scores (right) of the Ki-67 staining in tumors of mice treated with saline or peptide 17 . ${ }^{* *} \mathrm{P}<0.01$. Scale bar, $20 \mu \mathrm{m}$. (C) Representative images (left) and quantification scores (right) of the phospho-Akt (Ser473) in tumors of mice treated with saline or peptide 17 . $^{* * *} \mathrm{P}<0.001$. Scale bar, $20 \mu \mathrm{m}$. OC, ovarian cancer.

a YAP inhibitor, exerted a significant inhibitory effect on OC progression. Thus, the findings of the present study emphasized that targeting YAP suppresses OC progression through regulation of the $\mathrm{PI} 3 \mathrm{~K} / \mathrm{Akt} / \mathrm{mTOR}$ pathway, indicating the potential clinical value of peptide 17 in OC treatment.

The Hippo/YAP pathway plays a crucial role in development, growth and organogenesis, and dysregulation of this pathway has been associated with tumor progression $(15,16)$. Some studies have also found that the Hippo/YAP pathway may be involved in the initiation and progression of ovarian cancer $(10,17)$. Our study further indicated that targeting YAP with siRNA or YAP inhibitor significantly attenuated OC progression in vitro and in vivo through reducing activation of the PI3K/Akt/mTOR pathway. Although the connection between YAP and the PI3K/AKT/mTOR pathway has previously been demonstrated in other cancers $(18,19)$, these results of the present study depicted the regulation between YAP and the PI3K/AKT/mTOR pathway in OC. 
D

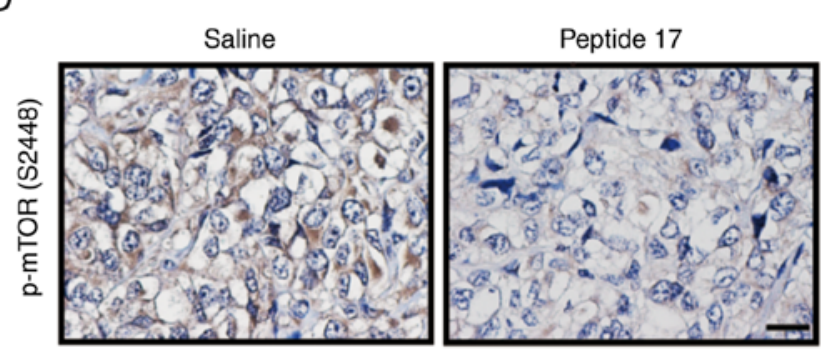

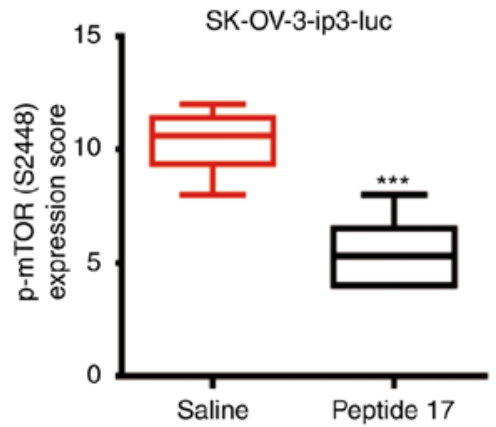

$\mathrm{E}$
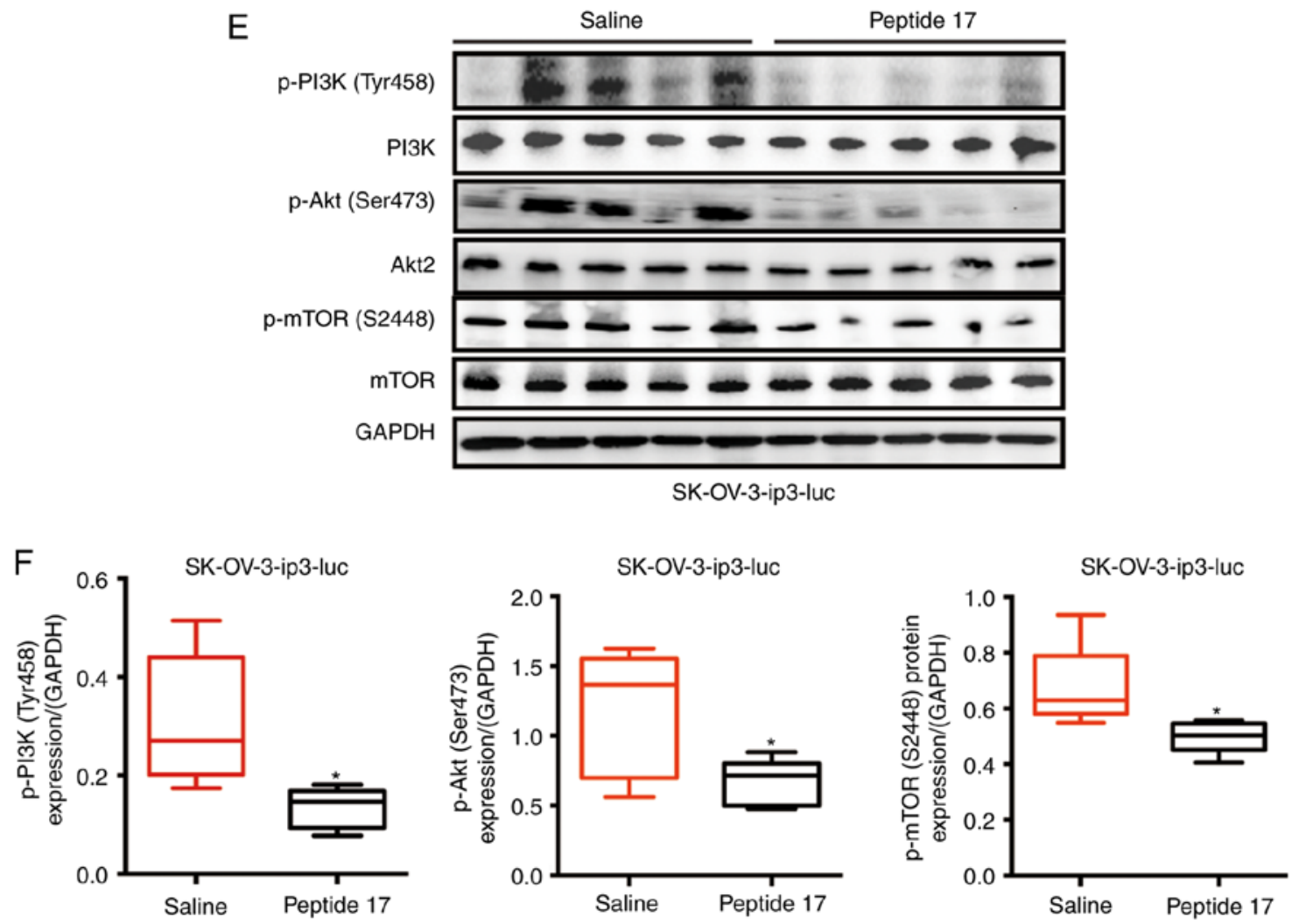

Figure 5. Continued. (D) Representative images (left) and quantification scores (right) of the phospho-mTOR (S2448) in tumors of mice treated with saline or peptide 17. ${ }^{* * *} \mathrm{P}<0.001$. Scale bar, $20 \mu \mathrm{m}$. (E) Western blot analysis of the expression of phospho-PI3K (Tyr458), PI3K, phospho-Akt (Ser473), Akt2, phospho-mTOR (S2448) and mTOR of tumors of mice treated with saline or peptide 17 and the quantification (F) ${ }^{*} \mathrm{P}<0.05$. OC, ovarian cancer.

Currently, therapy with small molecule inhibitors is an important therapeutic strategy for tumor progression. For example, based on the key role of poly [adenosine diphosphate (ADP)-ribose] polymerase (PARP) in DNA damage repair, PARP inhibitors have been approved by the U.S. Food and Drug Administration as monotherapy for patients with several different types of cancer $(20,21)$. In addition, lung cancers with activating EGFR mutations exhibit a notable response to treatment with EGFR tyrosine kinase inhibitors (22). With stronger binding affinity to TEAD1 rather than the YAP protein, peptide 17 is identified as a YAP-TEAD protein-protein interaction inhibitor (23), which can attenuate the oncogenic function of YAP and may thus be of value in the treatment of YAP-involved cancers $(23,24)$. In the present study, it was confirmed that peptide 17 markedly attenuated OC progression both in vitro and in vivo. As a small molecular inhibitor, peptide 17 exhibited strong potential for OC therapy in the clinical setting. Further experiments should be performed to investigate the safety of peptide 17 .
In summary, these findings of the present study highlighted the effectiveness of targeting YAP in restraining OC progression, and revealed that the underlying mechanism may be regulation of the PI3K/Akt/mTOR pathway. Of note, peptide 17 may be recommended as a therapeutic strategy against $\mathrm{OC}$ progression, providing novel options for $\mathrm{OC}$ management.

\section{Acknowledgements}

Not applicable.

\section{Funding}

This study was supported by a grant from the '973' Program of China (grant no. 2015CB553903 to Ding Ma and Junbo Hu), the National Science-Technology Supporting Plan Projects (grant no. 2015BAI13B05), the National Key Research and Development Program of China (grant no. 2016YFC0902901), 
the National Science Foundation of China (grant nos. 81372801 and 81572570), the National Science and Technology Major Sub-Project (grant no. 2018ZX10301402-002), and the Technical Innovation Special Project of Hubei Province (grant no. 2018ACA138). These grants provided financial supports for the study but did not impose restrictions on the design of the study, collection, analysis and interpretation of data, or writing of the manuscript.

\section{Availability of data and materials}

The datasets used during the present study are available from the corresponding author upon reasonable request.

\section{Authors' contributions}

XW conceived and designed the experiments. YJ, HL, JM, QH and YM performed the experiments. CS provided assistance with statistical analyses. XL, SX and XY conducted the bioinformatics analysis. XW and QG wrote the paper. ZY and TJ provided assistance with revising the manuscript. All authors have read and approved the final version of the manuscript.

\section{Ethics approval and consent to participate}

Human tissues were donated for research purposes by patients undergoing surgery at the Department of Obstetrics and Gynecology, Tongji Hospital, Huazhong University of Science and Technology, after obtaining written informed consent of the patients and the authorization of the Ethics Committee of Tongji Hospital (TJ-IRB20181103). All mouse experiments were conducted in accordance with a protocol approved by the Ethics Committee of Tongji Hospital, Tongji Medical College, Huazhong University of Science and Technology (Institutional Review Board Approval of Experimental Animals: IRB ID: TJ-A20160105).

\section{Patient consent for publication}

Not applicable.

\section{Competing interests}

The authors declare that they have no financial or non-financial competing interests.

\section{References}

1. Jayson GC, Kohn EC, Kitchener HC and Ledermann JA: Ovarian cancer. Lancet 384: 1376-1388, 2014.

2. Lengyel E: Ovarian cancer development and metastasis. Am J Pathol 177: 1053-1064, 2010.

3. Schwartz PE: Current diagnosis and treatment modalities for ovarian cancer. Cancer Treat Res 107: 99-118, 2002.

4. Basu-Roy U,Bayin NS, Rattanakorn K, Han E,Placantonakis DG, Mansukhani A and Basilico C: Sox2 antagonizes the Hippo pathway to maintain stemness in cancer cells. Nat Commun 6: $6411,2015$.
5. Harvey KF, Zhang $X$ and Thomas DM: The Hippo pathway and human cancer. Nat Rev Cancer 13: 246-257, 2013.

6. Liu AM, Xu MZ, Chen J, Poon RT and Luk JM: Targeting YAP and Hippo signaling pathway in liver cancer. Expert Opin Ther Targets 14: 855-868, 2010.

7. Pegoraro S, Ros G, Ciani Y, Sgarra R, Piazza S and Manfioletti G: A novel HMGA1-CCNE2-YAP axis regulates breast cancer aggressiveness. Oncotarget 6: 19087-19101, 2015.

8. Wu H, Wei L, Fan F, Ji S, Zhang S, Geng J, Hong L, Fan X, Chen Q, Tian J, et al: Integration of Hippo signalling and the unfolded protein response to restrain liver overgrowth and tumorigenesis. Nat Commun 6: 6239, 2015.

9. Gao Y, Zhang W, Han X, Li F, Wang X, Wang R, Fang Z, Tong X, Yao S, Li F, et al: YAP inhibits squamous transdifferentiation of Lkb1-deficient lung adenocarcinoma through ZEB2-dependent DNp63 repression. Nat Commun 5: 4629, 2014.

10. Zhang X, George J, Deb S, Degoutin JL, Takano EA, Fox SB, Bowtell DD, Harvey KF and AOCS Study group: The Hippo pathway transcriptional co-activator, YAP, is an ovarian cancer oncogene. Oncogene 30: 2810-2822, 2011.

11. He C, Lv X, Hua G, Lele SM, Remmenga S, Dong J, Davis JS and Wang C: YAP forms autocrine loops with the ERBB pathway to regulate ovarian cancer initiation and progression. Oncogene 34 : 6040-6054, 2015.

12. Wei X, Liu Y, Gong C, Ji T, Zhou X, Zhang T, Wan D, Xu S, Jin P, Yang X, et al: Targeting leptin as a therapeutic strategy against ovarian cancer peritoneal metastasis. Anticancer Agents Med Chem 17: 1093-1101, 2017.

13. Ji T, Gong D, Han Z, Wei X, Yan Y, Ye F, Ding W, Wang J, Xia X, Li F, et al: Abrogation of constitutive Stat 3 activity circumvents cisplatin resistant ovarian cancer. Cancer Lett 341: 231-239, 2013.

14. Gyorffy B, Lanczky A and Szallasi Z: Implementing an online tool for genome-wide validation of survival-associated biomarkers in ovarian-cancer using microarray data from 1287 patients. Endocr Relat Cancer 19: 197-208, 2012.

15. Wang Y, Pan P, Wang Z, Zhang Y, Xie P, Geng D, Jiang Y, Yu R and Zhou X: $\beta$-catenin-mediated YAP signaling promotes human glioma growth. J Exp Clin Cancer Res 36: 136, 2017.

16. Ma Y, Yang Y, Wang F, Wei Q and Qin H: Hippo-YAP signaling pathway: A new paradigm for cancer therapy. Int J Cancer 137: 2275-2286, 2015.

17. Hua G, Lv X, He C, Remmenga SW, Rodabough KJ, Dong J, Yang L, Lele SM, Yang P, Zhou J, et al: YAP induces high-grade serous carcinoma in fallopian tube secretory epithelial cells. Oncogene 35: 2247-2265, 2016.

18. Wang EY, Cheng JC, Thakur A, Yi Y, Tsai SH and Hoodless PA: YAP transcriptionally regulates ErbB2 to promote liver cell proliferation. Biochim Biophys Acta Gene Regul Mech: Jul 17, 2018 (Epub ahead of print) doi: 10.1016/j.bbagrm.2018.07.004.

19. Liu M, Lin Y, Zhang XC, Tan YH, Yao YL, Tan J, Zhang X, Cui YH, Liu X, Wang Y and Bian XW: Phosphorylated mTOR and YAP serve as prognostic markers and therapeutic targets in gliomas. Lab Invest 97: 1354-1363, 2017.

20. Bryant HE, Schultz N, Thomas HD, Parker KM, Flower D, Lopez E, Kyle S, Meuth M, Curtin NJ and Helleday T: Specific killing of BRCA2-deficient tumours with inhibitors of poly(ADP-ribose) polymerase. Nature 434: 913-917, 2005.

21. TuttA,RobsonM,GarberJE,DomchekSM,AudehMW,WeitzelJN, Friedlander M, Arun B, Loman N, Schmutzler RK, et al: Oral poly(ADP-ribose) polymerase inhibitor olaparib in patients with BRCA1 or BRCA2 mutations and advanced breast cancer: A proof-of-concept trial. Lancet 376: 235-244, 2010.

22. Dowell JE and Minna JD: EGFR mutations and molecularly targeted therapy: A new era in the treatment of lung cancer. Nat Clin Prac Oncol 3: 170-171, 2006.

23. Zhang Z, Lin Z, Zhou Z, Shen HC, Yan SF, Mayweg AV, Xu Z, Qin N, Wong JC, Zhang Z, et al: Structure-Based design and synthesis of potent cyclic peptides inhibiting the YAP-TEAD protein-protein interaction. ACS Med Chem Lett 5: 993-998, 2014.

24. Zhou Z, Hu T, Xu Z, Lin Z, Zhang Z, Feng T, Zhu L, Rong Y, Shen H, Luk JM, et al: Targeting Hippo pathway by specific interruption of YAP-TEAD interaction using cyclic YAP-like peptides. FASEB J 29: 724-732, 2015. 